\title{
(6) Developing person-centred analysis OPEN ACCESS \\ of harm in a paediatric hospital: a quality improvement report
}

\author{
Peter Lachman, Lynette Linkson, Trish Evans, Henning Clausen, \\ Daljit Hothi
}

Great Ormond Street Hospital for Children NHS Foundation Trust, London, UK

\section{Correspondence to} Dr Peter Lachman, Great Ormond Street Hospital for Children NHS Foundation Trust, London, WC1N 3JH, UK; Peter.Lachman@gosh.nhs.uk

Received 16 November 2014 Revised 12 February 2015 Accepted 19 February 2015 Published Online First 30 March 2015

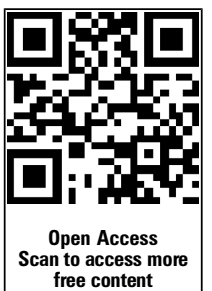

CrossMark

To cite: Lachman $P$, Linkson L, Evans T, et al. BMJ Qual Saf 2015;24:337-344.

\section{ABSTRACT}

The provision of safe care is complex and difficult to achieve. Awareness of what happens in real time is one of the ways to develop a safe system within a culture of safety. At Great Ormond Street Hospital, we developed and tested a tool specifically designed for patients and families to report harm, with the aim of raising awareness and opportunities for staff to continually improve and provide safe care. Over a 10-month period, we developed processes to report harm. We used the Model for Improvement and multiple Plan, Do, Study, Act cycles for testing. We measured changes using culture surveys as well as analysis of the reports. The tool was tested in different formats and moved from a provider centric to a person-centred tool analysed in real time. An independent person working with the families was best placed to support reporting. Immediate feedback to families was managed by senior staff, and provided the opportunity for clarification, transparency and apologies. Feedback to staff provided learning opportunities. Improvements in culture climate and staff reporting were noted in the short term. The integration of patient involvement in safety monitoring systems is essential to achieve safety. The high number of newly identified 'near-misses' and 'critical incidents' by families demonstrated an underestimation of potentially harmful events. This testing and introduction of a self-reporting, real-time bedside tool has led to active engagement with families and patients and raised situation awareness. We believe that this will lead to improved and safer care in the longer term.

Place the quality of patient care, especially patient safety, above all other aims.

Engage, empower, and hear patients and carers at all times.

Hear the patient voice, at every level, even when that voice is a whisper.

'Don Berwick' ${ }^{1}$

\section{DEFINING 'THE PROBLEM'}

The determination of the actual harm that occurs in hospitals is not really known. Voluntary reporting methods are biased by differences in understanding what constitutes harm and a focus on serious events. Under-reporting of adverse events and harm is common practice. $^{2}{ }^{3}$ It has been reported that $10 \%$ of UK adult patients admitted to hospital experienced harm, half of which were judged to be preventable. ${ }^{4}$ In paediatrics, reported harm in UK hospitals, as detected by the Paediatric Trigger Tool, is $14 \% .^{5}$ These reports are examples of studies to determine the scale of the problem from the view of healthcare providers. Patient-reported harm is different from that reported by clinicians. We believe that patients and families have different perspectives on harm and are witness to harm not seen by health professionals. $^{67}$

\section{THE FUTURE VISION}

In order to develop and sustain a safe healthcare system in which safety is the primary focus, an understanding of the nature of harm is essential. Personcentred care requires a focused system in which the voice of the people who receive care is heard and their understanding of harm is accepted and reported. This will enrich the professionals' understanding of harm.

Vincent et $a l^{8}$ suggest a professional's framework for measuring safety (table 1). Hollnagel defines safety as the sum of accidents that did not happen. ${ }^{9}$ Few systems measure 'near-misses'. 'What matters to patients' may not be the same as 'what matters to clinicians'. Safe care should be viewed from the perspective of 
Table 1 Framework for measurement of patient safety ${ }^{7}$

\begin{tabular}{ll}
\hline $\begin{array}{l}\text { Domain } \\
\text { Past harm }\end{array}$ & $\begin{array}{l}\text { Question to ask } \\
\text { Reliability }\end{array}$ \\
$\begin{array}{ll}\text { Has patient care been safe in the past? } \\
\text { Sensitivity to operations }\end{array}$ & $\begin{array}{l}\text { Is care safe today? } \\
\text { Anticipation and } \\
\text { preparedness }\end{array}$ \\
$\begin{array}{l}\text { Integration and learning } \\
\text { Till care be safe in the future? }\end{array}$ \\
\hline
\end{tabular}

the patient and family as well as from that of the provider. ${ }^{10} 11$

In this paper, we present the development, introduction and subsequent learning and outcomes of a person-centred tool that has the potential to address some of the complex issues on patient safety from the viewpoint of the patient and family.

\section{BACKGROUND}

Great Ormond Street Hospital for Children NHS Foundation Trust is a tertiary and quaternary paediatric hospital with a strategic vision to achieve Zero Harm. The organisation's systems to detect harm include staff critical incident reporting through DATIX, ${ }^{12}$ a modified patient safety thermometer ${ }^{13} 14$ and a modified mortality review tool. ${ }^{15}$ In addition, specific harms are proactively measured through quality and safety projects. Parents' perceptions are reviewed through the complaints process and the Patient Advice and Liaison Service (PALS).

Through the detection of adverse events, numerous interventions have been introduced to mitigate against harm to patients. Accessing human factors and reliability theory, ${ }^{16}$ we have developed systems to increase consistency in care such as care bundles, standardised processes and safety huddles. We have focused on components of patient safety harm as perceived by professionals. We believe that for a more holistic approach, we also need a process that identifies 'harm' perceived by patients and their families.

\section{AIM}

The primary aim of this study was to build a simple, user-friendly system that would allow us access to the patient and their family's perception of 'harm'. As a secondary aim, we hypothesised that by developing a process for patients and their families to proactively report harm, we would alter the clinical staff's perception of what constitutes harm and how best to manage risk or harm at the front line.

\section{METHODOLOGY}

\section{Study site}

The quality improvement project was conducted on a 14 bed renal inpatient ward from April 2013 to March 2014.

\section{Stakeholder involvement}

Stakeholder involvement was secured by consulting the risk and complaints team, PALS, medical staff, senior executives and patient forums including the Young Persons' Forum. A parent was an active and equal member of the project's planning and operational groups. The project manager was independent and external to the ward. We developed an escalation policy for the reporting system to align it with in-house governance and safeguarding structures. We designed and tested an information sheet to accompany the written consent form.

\section{Baseline measurement of safety culture}

We measured baseline safety climate using the Manchester Patient Safety Framework (MaPSaF) tool. ${ }^{17}$ A Safety Climate survey ${ }^{18}$ was completed electronically by permanent ward nursing staff, excluding transient bank staff who ordinarily did not attend staff meetings, and who covered up to 13 shifts a month. Both measurements were completed at the start of the study and again at the end of the study. We could not guarantee that the same staff members were present at the prestudy and poststudy assessments. We accepted this bias as our objective was to measure the overall aggregate culture.

\section{ETHICS}

Ethics approval was obtained via the Integrated Research Application System and was approved by the Brent Research Ethics Service Committee. The committee raised concerns about how parental reporting may impact the care of their children and how we would manage the adverse consequences of reporting harm. With the parent advisor's support, we designed systems to ensure that children and parents were adequately supported through access to members of ward staff, PALS and the risk team.

\section{DEVELOPMENT}

There were three stages developing the reporting tool.

\section{Stage 1}

We tested parents' engagement, readiness and ability to report harm by adapting an existing Canadian DATIXbased questionnaire ${ }^{19}$ that families completed anonymously at the point of discharge. This questionnaire also provided families with the opportunity to give positive feedback. We completed over 20 Plan, Do, Study, Act $(\mathrm{PDSA})^{20}$ cycles over a 20 -week period to test the relevance, application and value of the questionnaire and the level of support required to complete the questionnaire. Our tests highlighted important limitations of this method. Families provided greater detail in their reports when supported, although they could still complete the questionnaire unaided. The provision of one opportunity to report caused a reporting bias towards events that occurred near discharge. Anonymous reporting limited 
the ability to investigate events and introduced a concern that we may misinterpret the 'risk'.

\section{Stage 2}

We designed a patient-centric process for managing risk that comprised three components. The first component is a simple, real-time, reporting tool designed to be completed by patients and their families daily (figure 1). The second component, on the reverse of the reporting tool, is the 'investigation and action' table to record the mandated timely discussion between a senior nurse and the family. This aims to achieve a better understanding of the harm and gain consensus on how the harm will be managed (figure 2). The final component is a centralised record of all the reported events, the outcome and subsequent actions (figure 3 ).

We used a series of PDSAs, codesign and codevelopment with staff and families to design and then test usability and acceptance by staff and families. Ten different versions of the tool were tested.

\section{Stage 3}

Once the design of the tool was completed, we tested implementation of the tool. Instruction posters were placed at the patient's bedside. We analysed family engagement with an external facilitator versus the staff approaching the family daily. A strap line was handwritten by the project manager on the daily day and night care plans to remind staff to check the reporting tool at the end of their shift. Staff members were engaged on a 1:1 basis to be made aware of tests of change and how to manage reports made by families/patients. Once the process had been tested on a selected cohort of patients, it was spread to the entire ward.

\section{RESULTS}

Usability and acceptance of reporting harm

Patients and families were able to understand and use the questionnaire in stage 1 but preferred the daily reporting tool developed in stage 2 . The questionnaire was time-consuming and reliant on understanding English and thus excluded some of the multiethnic patients. There was a reluctance to complete the questionnaire on readmissions. In comparison, the simplicity of the daily tool and use of pictures enabled reporting by all families and patients, across language barriers. It was also child friendly, such that a 12 -year-old patient independently reported harm. The questionnaire offered families the opportunity to report positive experiences, which was not possible with the daily tool.

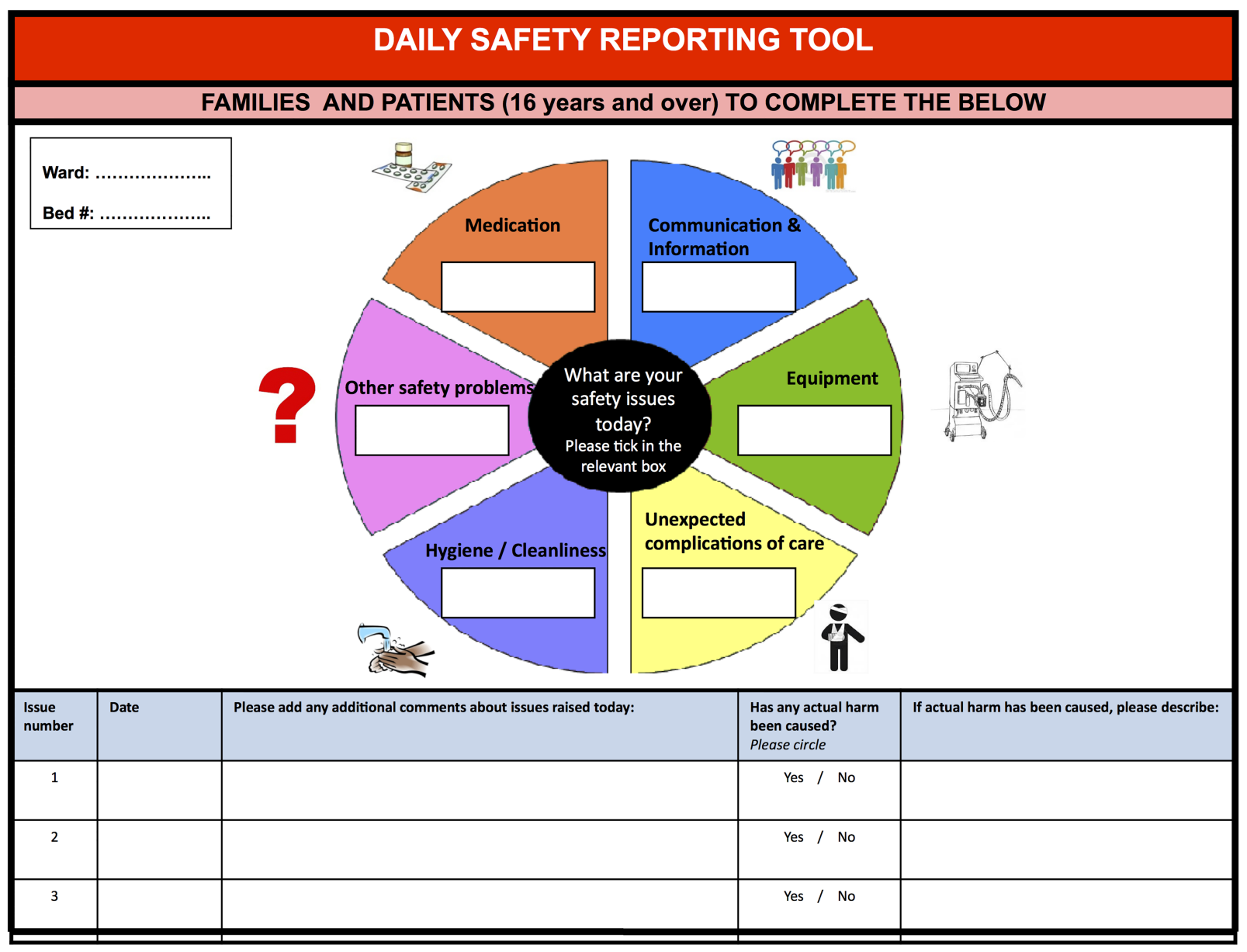

Figure 1 Daily safety reporting tool. 
- Staff member to discuss the patient safety issue with family.

STAFF MEMBER TO COMPLETE THE BELOW

ANALYSIS OF DAILY PATIENT SAFETY ISSUES

Staff member to review and tick the most appropriate categories (below) that describes the patient safety issue.

Ensure that the issue number listed by families is coordinated with the issue number listed on the category section.

Staff member to write a brief description of the actions taken to resolve patient safety issues raised.
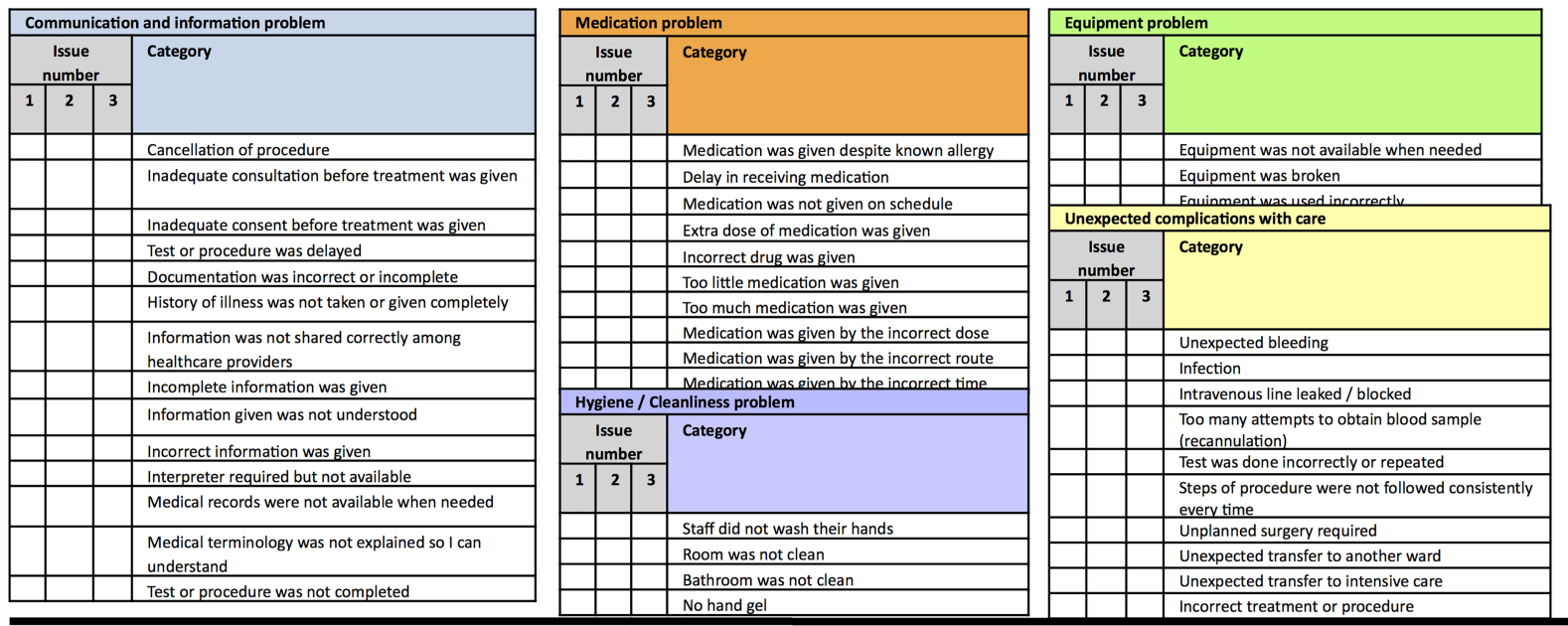

Actions taken to resolve patient safety issues raised (to be completed by staff):

Issue 1) ...

Issue 2)

Issue 3)

Figure 2 Analysis of daily patient safety issues.

The application of the 'daily tool' led to families successfully reporting incidents supported by the project manager. During the 'daily tool' study period, we also gave families the option to complete the previous questionnaire, anonymously at the point of discharge. Seventeen families were approached, of which three chose to use the electronic version, with two safety concerns being recorded.

\section{Reporting of harm by parents and patients}

During the stage 1, 22-week 'questionnaire' study period, 100 families were approached and 85 participated. One family proactively approached the project team before being introduced to the questionnaire and asked to report an event. Families successfully reported harms, but the nature of the harms differed from staff reporting. Families reported a higher proportion of 'minor' degree harms and 'near-miss' events (table 2). The highest proportion of safety concerns was classified as miscommunication, while eight 'other' events related to significant delays to inpatient care or problems related to cleanliness and hygiene. During the same observation period, routine staff reporting of critical incidents through DATIX increased significantly by $67 \%$ from 1.29 to 2.05 reports per week $(\mathrm{p}<0.05)$. Only $3 \%$ of the incidents

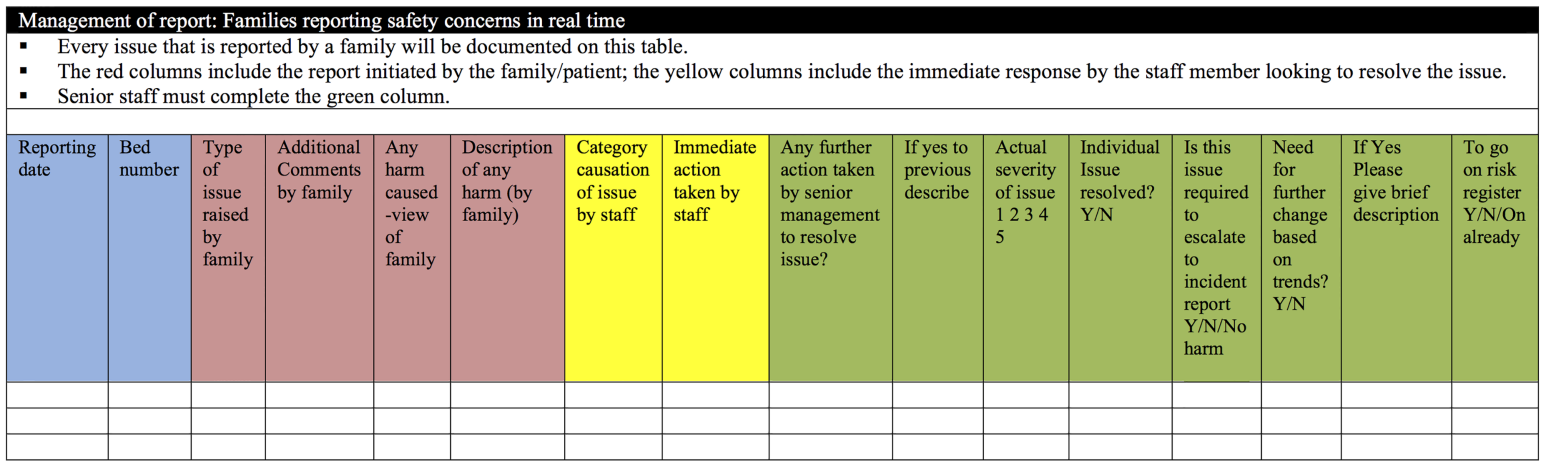

Figure 3 Management of report: families reporting safety concerns in real time. 
Table 2 Types of safety concerns reported by families and patients using different questionnaire designs

\begin{tabular}{llc}
\hline $\begin{array}{l}\text { Type of safety } \\
\text { concern }\end{array}$ & $\begin{array}{l}\text { Predischarge } \\
\text { electronic } \\
\text { questionnaire (\%) }\end{array}$ & $\begin{array}{l}\text { Daily paper-based } \\
\text { questionnaire (\%) }\end{array}$ \\
\hline $\begin{array}{l}\text { Communication } \\
\text { Medication }\end{array}$ & 31 & 30 \\
Equipment use & 21 & 3 \\
Complication of care & 15 & 33 \\
Hygiene/cleanliness & 24 & 7 \\
and 'others' & & 27 \\
Total & 100 & 100 \\
\hline
\end{tabular}

reported by families were repeated in the staff reporting processes.

There was a slight shift in the type of events reported by families between the methods described. The reporting by families decreased when staff members directly involved in the child's care facilitated the reporting process.

During the second 13-week, 'daily tool' study period, 30 events were recorded, the highest proportion almost equally relating to equipment problems and communication issues (table 2). During this study period, staff critical incident reporting increased to 2.31 reports per week.

\section{Ward safety culture}

To assess the impact of families reporting harm on the safety culture in the clinical area and changes in staff members' perceptions on what constitutes harm, we measured the culture of safety using two methods. The MaPSaF tool ${ }^{17}$ was completed during a facilitated session on two occasions with the nurses. The project manager facilitated each session and each group discussed on topics such as teamwork and communication (table 3). Individual and group scores for the framework were obtained. We did not seek to infer statistical significance, as this is not appropriate for the MaPSAF. All staff also completed the Safety Climate score, to provide us with an indication of the climate of safety on the ward.

All professional groups rated the organisation and the ward safety culture as having systems in place to deal with harm. Ward nurses believed that their team had a more proactive stance to patient safety and the consultants and junior nurses rated their safety culture as system based. The consultants believed that the organisation always looks for potential harm, but the remaining groups considered the organisation to be less developed. Most groups rated their team and the organisation as being proactive for system failures and individual responsibility. All teams thought that they were proactive for learning and effecting change.

The results of the $\mathrm{MaPSaF}^{17}$ (completed by the nurses) and Safety Climate $^{18}$ tools did not show a significant change in scores over the study period (tables 3 and 4). The clinical staff members' perception of a safety climate remained largely unchanged, though completion rates of the climate tool had declined over the observation period from 58\% (29/ $50)$ to $38 \%(20 / 53)$. The reflections of members of staff were suggestive of a positive change and all welcomed the concept of families reporting their concerns. When asked about communication concerning safety issues, three of the four groups answered that their team was proactive. One nursing group thought that for their team safety was integrated in the way they worked. Communication within the organisation about safety issues was varied. Two nursing groups thought that the organisation had systems in place while the consultants thought that the organisation was proactive.

Table 3 MaPSaF results

\begin{tabular}{|c|c|c|c|c|c|}
\hline $\begin{array}{l}\text { A Pathological } \\
\text { B Reactive } \\
\text { C Systems in place } \\
\text { D Proactive } \\
\text { E Generative }\end{array}$ & $\begin{array}{l}\text { Junior Nurse } \\
\text { May } 2013\end{array}$ & $\begin{array}{l}\text { Junior Nurse } \\
\text { January } 2014\end{array}$ & $\begin{array}{l}\text { Senior Nurse } \\
\text { May } 2013\end{array}$ & $\begin{array}{l}\text { Senior Nurse } \\
\text { January } 2014\end{array}$ & $\begin{array}{l}\text { Consultants } \\
\text { January } 2014 \\
\text { Applied once }\end{array}$ \\
\hline Number completed & 18 & 18 & 10 & 13 & 5 \\
\hline Number of staff & 26 & 28 & 14 & 15 & 10 \\
\hline Percentage completed & 69 & 64 & 71 & 86 & 50 \\
\hline Priority given to safety & C & E $\boldsymbol{\Delta}$ & $\mathrm{D}$ & D & $\mathrm{C}$ \\
\hline System errors and individual responsibility & C & E $\boldsymbol{\Delta}$ & $\mathrm{D}$ & D & $\mathrm{D}$ \\
\hline Learning and effective change & D & D & D & D & $\mathrm{D}$ \\
\hline Communication about safety issues & $\mathrm{D}$ & D & $\mathrm{D} / \mathrm{E}$ & $C \nabla$ & D \\
\hline \multicolumn{6}{|l|}{ Organisation } \\
\hline Priority given to safety & $\mathrm{D}$ & D & $\mathrm{D}$ & $C \nabla$ & $\mathrm{E}$ \\
\hline System errors and individual responsibility & D & D & $\mathrm{C}$ & E $\boldsymbol{\Delta}$ & $\mathrm{D}$ \\
\hline Learning and effective change & $\mathrm{E}$ & $\mathrm{D} \boldsymbol{\nabla}$ & $\mathrm{D}$ & $C \nabla$ & D \\
\hline Communication about safety issues & $\mathrm{E}$ & $\mathrm{D} \boldsymbol{\nabla}$ & $\mathrm{C}$ & $B \boldsymbol{\nabla}$ & $\mathrm{D}$ \\
\hline
\end{tabular}

MaPSaF, Manchester Patient Safety Framework; $\mathbf{\Lambda}$, move up MaPSaF scale; $\mathbf{\nabla}$, move down MaPSaF scale. 


\begin{tabular}{lr}
\hline & $\begin{array}{l}\text { January } \begin{array}{c}2014 \\
\text { staff }\end{array} \\
20 / 50 / 53 \text { staff }\end{array}$ \\
\hline Overall, mean score* & 4.41 \\
Overall safety climate mean score & 4.46 \\
Aggregate percentage of respondents reporting a positive safety climate with an average score of 4 or above & 4.59 \\
\hline
\end{tabular}

*The average of all the questions answered within the survey to give an indication of the overall perception of safety in the clinical area (1—strongly disagree, 5-strongly agree).

\section{DISCUSSION}

There is a clear need for healthcare providers to seek ways to hear the voice of the patient and families and how they experience harm. ${ }^{21}$ Patients have varying attitudes as to their ability to discuss patient safety and one cannot assume that all are ready to participate in the patient safety processes. ${ }^{22}$ The development of methods to facilitate the voice of patients and families to be heard has had different levels of success. A review of published research on patient reporting indicates that there is no uniform way to elicit the experience of patients and their families, ${ }^{10}$ though patients are able to report harm and safety issues, if given the opportunity.

It may be difficult for healthcare providers to align their understanding of harm with that of patients and their families. The views of hospital staff differ to that of families. A Patient Measure of Safety has been tested in an adult hospital setting with some success. $^{23-25}$ This was similar to the process followed in stage 1 in this study with comparable safety themes. We found that parents report vulnerability when their children are admitted to a hospital ward. They have to relinquish a degree of control to the professionals involved in their child's care. The opportunity to 'protect' their child from harm is instinctive and therefore we believe that the daily opportunity to report their experiences in terms of safety adds a new dimension to the development of mindfulness in the clinical environment. There is a delicate balance between family empowerment and vulnerability when developing processes involving families in reporting harm in clinical environments. It is possible that asking patients and families to report harm may affect the health provider-patient relationship. ${ }^{26}$ When the ward staff facilitated or supported reporting events were not reported, which could suggest that families felt uncomfortable reporting harm directly to clinical staff involved in their child's care.

The families in this study responded favourably to the opportunity to feedback positive aspects of their admission to the clinical staff. One explanation is that families may hold the belief that this helped to counteract possible negative repercussions from the family reporting safety concerns. Likewise, the positive feedback was valued by the ward staff and was conceivably an enabler in their engagement with the study.
Both families and ward staff stated a preference for the daily tool over the questionnaire. The families preferred the simplicity and the ability to report repeatedly. This may be related to personal preferences and the additional component of anonymity. For the staff, the tool was one component of the 'harm that matters to me' process that effectively allows mitigation against harm. It also became a gateway to other opportunities, such as bridging communication with families, transparency and opening discussions with ward staff for learning and reflection. Our results indicated an increased reporting of events by nursing staff, more transparency in the discussion of harm and greater willingness of the ward staff to discuss safety events openly. Finally, the 'harm that matters to me' reporting tool improved situation awareness for safety on the ward by staff and families alike. It may be that the increased reporting could be a Hawthorne effect or the result of an increased awareness of harm by staff members.

The measurement of safety climate is complex, ${ }^{27}$ our study period was short and thus measures of cultural change were unlikely to demonstrate significant change. One could argue that the response rate was not adequate to draw conclusions; however, culture change requires a more longitudinal study. Nonetheless, there was unanimous support for the 'harm that matters to me' approach in enhancing the safety climate.

\section{LIMITATIONS}

Initially, patients were selected for testing and thus we may have an exaggerated positive response to reporting. Weekend admissions may put patients at higher risk of adverse events compared with planned weekday admissions, ${ }^{28}$ but we did not have the resources to enable reporting over weekends or evenings. The renal team is well known to many of the families who are admitted to the ward and this relationship may have influenced family engagement with the study and biased reporting. The high baseline score and possible insensitivity of the $\mathrm{MaPSaF}^{17}$ and Safety Climate ${ }^{18}$ tools for detecting subtle changes could explain the apparent lack of difference in safety climate. A simple questionnaire or interview may have been more informative. The sample size may appear to be small but as this is a quality improvement project, we believe that this does not detract from the findings. 


\section{CONCLUSION AND NEXT STEPS}

In the quest for improved patient safety, healthcare providers need to include the patients as equal partners in the detection of harm. Vincent and Davis ${ }^{29}$ call for the integration of patient involvement in safety monitoring systems. This is imperative if one is to apply a proactive approach to introduction of the framework for the measurement of patient safety. ${ }^{8}$ The introduction of a simple, real-time bedside reporting tool and risk management process facilitated active engagement with families and patients, and the opportunity for disclosure and learning for staff.

We recommend that units consider ways to move from a reactive approach once harm has occurred, to a proactive assessment using the real experts, the patients and their families. The tool is simple and effective. It will require a change of culture and approach on the ward, but that is possible when safety becomes a priority.

We believe that the introduction of this simple family and person-centred tool will raise awareness and help healthcare providers to know what is really happening in real time. This, in turn, will build resilience and radically change the dynamics of patient safety in healthcare. It is not intended to determine whether a clinical area is safe as no one method can serve that purpose. Rather, it is intended to add to the tapestry of measures that provide an overview of the safety of a clinical area and enhance the culture of inclusion. The next step will be to test the tool in a wider setting to correlate its introduction with an improved culture of safety and decrease in harm.

Twitter Follow Peter Lachman at @peterlachman

Acknowledgements We thank all families and hospital members of staff on Eagle Ward, Great Ormond Street Hospital for Children NHS Foundation Trust who participated in this study. Charlotte Magness, Project Manager and Andrew Henderson, parent, project advisor and lay member of the steering group made invaluable contributions to the project. Beki Moult proofread the final manuscript.

Contributors PL was the project supervisor; co-conceptualised and designed the study, drafted the initial and final manuscripts and approved the final manuscript as submitted. LL: implemented and developed the study; then reviewed and approved the final manuscript as submitted. TE was the front line team lead and implemented the study; then reviewed and approved the final manuscript as submitted. HC:

coconceptualised the study and was the project lead, assisted in early drafts, then reviewed and approved the final manuscript as submitted. DH was the clinical supervisor for the project and coauthored the manuscript with PL and approved the final manuscript as submitted. All authors contributed to the analysis of the data, and the interpretation of the results. All authors have contributed to and approved the revised version.

Funding This study was funded by The Health Foundation's SHINE 2012 programme (http://www.health.org.uk)

\section{Competing interests None.}

Ethics approval Brent Research Ethics Service Committee.

Provenance and peer review Not commissioned; externally peer reviewed.
Data sharing statement All data are presented in this paper.

Open Access This is an Open Access article distributed in accordance with the Creative Commons Attribution Non Commercial (CC BY-NC 4.0) license, which permits others to distribute, remix, adapt, build upon this work non-

commercially, and license their derivative works on different terms, provided the original work is properly cited and the use is non-commercial. See: http://creativecommons.org/licenses/by$\mathrm{nc} / 4.0 /$

\section{REFERENCES}

1 National Advisory Group on the Safety of Patients in England. A Promise to Learn-a Commitment to Act: Improving the Safety of Patients in England. 2013. https://www.gov.uk/ government/uploads/system/uploads/attachment_data/file/ 226703/Berwick_Report.pdf (accessed 6 Jul 2014).

2 Kingston MJ, Evans SM, Smith BJ, et al. Attitudes of doctors and nurses towards incident reporting: a qualitative analysis. Med J Aust 2004;181:36-9.

3 Hooper AJ, Tibballs J. Comparison of a Trigger Tool and voluntary reporting to identify adverse events in a paediatric intensive care unit. Anaesth Intensive Care 2014;42:199-206.

4 Vincent C, Aylin P, Franklin Bryony D, et al. Is health care getting safer? BMJ 2008;337:a2426.

5 Chapman SM, Fitzsimons J, Davey N, et al. Prevalence and severity of patient harm in a sample of UK-hospitalised children detected by the Paediatric Trigger Tool. BMJ Open 2014;4:e005066. (accessed 12 Aug 2014).

6 Weissman JS, Schneider EC, Weingart SN, et al. Comparing patient-reported hospital adverse events with medical record review: do patients know something that hospitals do not? Ann Intern Med 2008;149:100-8.

7 Weingart SN, Pagovich O, Sands DZ, et al. What can hospitalized patients tell us about adverse events? Learning from patient-reported incidents. J Gen Int Med 2005;20:830-6.

8 Vincent C, Burnett S, Carthey J. Safety measurement and monitoring in healthcare: a framework to guide clinical teams and healthcare organisations in maintaining safety. BMJ Qual Saf 2014;23:670-7.

9 Hollnagel E. Safety-I and Safety-II: the past and future of safety management. London: Ashgate, 2014.

10 Ward JK, Armitage G. Can patients report patient safety incidents in a hospital setting? A systematic review. BMJ Qual Saf 2012;21:685-99.

11 King A, Daniels J, Lim J, et al. Time to listen: a review of methods to solicit patient reports of adverse events. Qual Saf Health Care 2010;19:148-57.

12 DATIX. http://www.datix.co.uk (accessed 30 Jan 2015).

13 Power M, Stewart K, Brotherton A. What is the NHS Safety Thermometer? Clinical Risk 2012;18:163-9.

14 Van Ree J, Hussein R, Hothi DK. Measuring Composite Harm on a Paediatric Ward......... a journey to Harm Free Care. Abstract. International Forum on Quality \& Safety in Healthcare 2013, London UK.

15 Whittington J, Simmonds T, Jacobsen D. Reducing Hospital Mortality Rates (Part 2). IHI innovation series whitepaper. Cambridge, Massachusetts: Institute for Healthcare Improvement. http://www.ihi.org/resources/Pages/IHIWhitePapers/Reducing HospitalMortalityRatesPart2.aspx (accessed 20 Jan 2015).

16 Weick K, Sutcliffe K. Managing the unexpected. Assuring high performance in an age of complexity. San Francisco: Jossey Bass, 2001. 
17 Manchester Patient Safety Framework (MaPSaF). 2006. http:// www.nrls.npsa.nhs.uk/resources/?EntryId45 =59796 (accessed 30 Jan 2015).

18 Sexton JB, Helmreich RL, Neilands TB. The Safety Attitudes Questionnaire: psychometric properties, benchmarking data, and emerging research. BMC Health Serv Res 2006;6:44-53.

19 Daniels JP, Hunc K, Cochrane D, et al. Identification by families of pediatric adverse events and near misses overlooked by health care providers. CMAJ 2012;184:29-34.

20 Berwick DM. A primer on leading the improvement of systems. BMJ 1996;312:619-22.

21 Francis R. Report of the Mid Staffordshire NHS Foundation Trust Public Inquiry: executive summary. TSO Shop, 2013.

22 Davis RE, Sevdalis N, Vincent CA. Patient involvement in patient safety: how willing are patients to participate? BMJ Qual Saf 2011;20:108-14.

23 Lawton R, McEachan RRC, Giles SJ, et al. Development of an evidence-based framework of factors contributing to patient safety incidents in hospital settings: a systematic review. BMJ Qual Saf 2012;21:369-80.
24 Giles SJ, Lawton RJ, Din I, et al. Developing a patient measure of safety (PMOS). BMJ Qual Saf 2013;22:554-62.

25 McEachan RRC, Lawton RJ, O'Hara JK, et al. Developing a reliable and valid patient measure of safety in hospitals (PMOS): a validation study. BMJ Qual Saf 2014;23: 565-73.

26 Hrisos S, Thomson R. Seeing it from both sides: do approaches to involving patients in improving their safety risk damaging the trust between patients and healthcare professionals? An interview study. PLoS One 2013. http://journals.plos.org/plosone/article?id=10.1371/journal. pone.0080759 (accessed 26 January 2015).

27 The Health Foundation. Measuring safety culture. London: The Health Foundation, 2011. http://www.health.org.uk/ publications/measuring-safety-culture/ (accessed 31 Jan 2015).

28 Aylin P, Yunus A, Bottle A, et al. Weekend mortality for emergency admissions. A large, multicentre study. Qual Saf Health Care 2010;19:213-17.

29 Vincent C, Davis R. Patients and families as safety experts. CMAJ 2012;184:15-16. 